\section{PSICOLOGIA IBEROAMERICANA}

\section{Psicología lberoamericana}

ISSN: 1405-0943

psicología.iberoamericana@uia.mx

Universidad Iberoamericana, Ciudad de

México

México

Herrera Harfuch, María Fernanda; Pacheco Murguía, María Paula; Palomar Lever, Joaquina; Zavala Andrade, Daniela

La Adicción a Facebook Relacionada con la Baja Autoestima, la Depresión y la Falta de Habilidades

Sociales

Psicología Iberoamericana, vol. 18, núm. 1, enero-junio, 2010, pp. 6-18

Universidad Iberoamericana, Ciudad de México

Distrito Federal, México

Disponible en: http://www.redalyc.org/articulo.oa?id=133915936002

Cómo citar el artículo

Número completo

- Más información del artículo

Página de la revista en redalyc.org

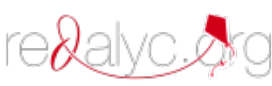

Sistema de Información Científica

Red de Revistas Científicas de América Latina, el Caribe, España y Portugal

Proyecto académico sin fines de lucro, desarrollado bajo la iniciativa de acceso abierto 


\title{
La Adicción a Facebook Relacionada con la Baja Autoestima, la Depresión y la Falta de Habilidades Sociales
}

\author{
Facebook Addiction Related to Low Self-Esteem, Depression \\ and Lack of Social Skills
}

María Fernanda Herrera Harfuch, María Paula Pacheco Murguía, Joaquina Palomar Lever, Daniela Zavala Andrade *

UnIVERSIDAD IBEROAMERICANA, CD. DE MÉXICO

\section{RESUMEN}

La presente investigación tuvo como objetivo encontrar si existía relación alguna entre la adicción a Facebook, la baja autoestima, la depresión y la falta de habilidades sociales. Fue un diseño no experimental, de tipo transversal. Se utilizó el cuestionario adaptado de Young (1998, citado en Navarro \& Jaimes, 2007), Fargues, Lusar, Jordania \& Sánchez (2009), Cándido, Saura, Méndez, e Hidalgo (2000), Yesavage (1986; citado en De la Vega, 2009) y García (1998). Participaron 63 alumnos de la Universidad Iberoamericana, de los cuales $60.3 \%$ eran mujeres y $39.7 \%$ hombres, y el mayor porcentaje de edad fue 22 años. El instrumento se complementó con variables sociodemográficas, el cuestionario de Adicción a Facebook para Jóvenes (de propia creación 2009), el Cuestionario de Evaluación de la Autoestima para Alumnos de Enseñanza Secundaria (García, 1998), el Cuestionario de Evaluación de Dificultades Interpersonales en la Adolescencia (Cándido, et al., 2000), la Escala de Depresión Geriátrica (Yesavage, 1986; citado en De la Vega, 2009), los Criterios de Diagnóstico de Adicción a internet (Young, 1998; citado en Navarro, 2007), y el Cuestionario de Experiencias Relacionadas con Internet (Fargues \& cols., 2009). Respecto de los resultados, se encontraron diferencias estadísticamente significativas entre los adictos y los no adictos a Facebook, lo que permitió ver que existe una relación estadísticamente significativa entre la adicción a Facebook y la baja autoestima, la depresión y la falta de habilidades sociales. A partir de ese descubrimiento se dedujo que los adictos a Facebook son más propensos a la depresión, a tener menos habilidades sociales y a mostrar una autoestima más baja.

Descriptores: Facebook, depresión, autoestima, habilidades sociales.

\begin{abstract}
This study had the purpose to find out if there was any relationship between Facebook's addiction, low self-esteem, depression and the lack of social-skills. It was an unexperimental design, of cross type. A questionnaire made by Young (1998, quoted in Navarro \& Jaimes, 2007), Beranuy et al. (2009), Candido et al. (2000), Yesavage (1986; quoted in De la Vega, 2009) and Garcia (1998) was adapted for its use in this investigation. There were 63 students of the "Universidad Iberoamericana" who participated, being $60.3 \%$ women and 39.7\% men (being 22 years-old the higher percentage). The instrument was

\footnotetext{
* Para correspondencia: María Paula Pacheco Murguía, Dirección: Colina del César \#7 Colinas de Tarango, C. P. 01610. Teléfono: 5643 9894, Celular: 044553237 8064, Correo electrónico: pach3203@hotmail.com. • Maria Fernanda Herrera Harfuch, Dirección: Monte de Funiar \#9 (frente al 60) Col. Jardines en la Montaña, C. P.: 14210. Teléfono: 5645 2061, Celular: 044558581 5387, Correo electrónico: nabeiu@hotmail.com. • Joaquina Palomar Lever, Universidad Iberoamericana-Ciudad de México, Prol. Paseo de La Reforma 880, C.P. 01219, Col. Lomas de Santa Fe, México, D.F., Tel. 59504000 Ext. 4866; correo electrónico: joaquina.palomar@uia.mx. • Daniela Zavala Andrade, Dirección: Hidalgo 18 casa 3 Col. Florida, C. P.: 01030. Teléfono: 66233 60, Celular: 044554140 5653, Correo electrónico: dzavalangc@hotmail.com.
} 
complemented by sociodemographic variables, and also by the questionnaire of Addiction to Facebook for Youths (of own creation 2009), the Questionnaire of Evaluation of High-school Students' Self-esteem (Garcia, 1998), the Questionnaire of Adolescent Interpersonal Difficulties Evaluation (Candid et al. 2000), the Scale of Geriatric Depression (Yesavage, 1986; quoted in De la Vega, 2009), the Addiction to Internet Diagnosis Criteria (Young, 1998, quoted in Navarro, 2007) and the Questionnaire of Experiences Related to Internet (Fargues et al., 2009).

Such as predicted, results reveal statistically significant differences among the groups of addicts and non-addicts to Facebook, therefore allowing to see there are statistically significant relations among Facebook's addiction and low self-esteem, depression and lack of social skills. It was concluded that Facebook's addicts are more prone to depression, to have less social abilities or skills and a lower self-esteem.

Keywords: Facebook, depression, self-esteem, social skills.

\section{INTRODUCCIÓN}

Todo organismo vivo debe integrar elementos y dinámicas de interacción emergentes para aumentar sus posibilidades de supervivencia. Así como este proceso de integración es necesario en el ámbito biológico, también la parte social es de suma importancia. En cuanto a las nuevas herramientas tecnológicas, el ser humano tiene que ir adaptándose a ellas en la medida en que éstas surgen. Estas tecnologías son conocidas como TIC (Tecnologías de Información y Comunicación), las cuales transforman la vida cotidiana de los sujetos cuando deben aplicarlas para enfrentar nuevos desafíos. Esto implica que el uso de las TIC es muy importante para el desarrollo individual y colectivo (Zermeño, Arellano \& Ramírez, 2005).

A pesar de que Internet surgió en Estados Unidos, su uso se ha expandido de manera exponencial y se ha convertido en una herramienta sumamente importante y popular en todo el mundo. En 1990 la mayor cantidad de usuarios de internet residía en dicho país. Cerca de $70 \%$ de la población mundial que utilizaba esta herramienta de trabajo y comunicación vivía en este país, y ya en 2005 se pudo constatar que este porcentaje disminuyó a $30 \%$ por cuestiones de migración (Raacke \& Bonds-Raacke, 2008). Internet ya no es un tema nuevo en nuestra época, y desde su surgimiento los científicos se han dedicado a estudiar cómo influye en nuestro comportamiento (Llamas, 2005).

Estos estudios han abarcado lo que es el fenómeno de internet en general, estudiándolo tanto como herramienta de trabajo así como instrumento de entretenimiento u ocio y de comunicación, el cual se ha convertido en un objeto de interés tan necesario para algunas personas que actualmente se puede encontrar el término "adicción a Internet" (Navarro \& Jaimes, 2007; Fargues, Lusar, Jordania \& Sánchez, 2009; Llamas, 2005; Jiménez \& Pantoja, 2007) en diferentes estudios, donde también se analizan las consecuencias del abuso en la utilización de este medio (Andreu,1999; Cruzado, Matos \& Kendall, 2006; Echeburua, 1999; Estalló, 2000a, 2000b, 2001; Goldberg, 1995; Luengo, 2004; Madrid, 2000; Niemz, Griffiths \& Banyard, 2005; Shapira, Lessign, Goldsmith, Szabo, Lazoritz \& Gold, 2003; Suhail \& Bargees, 2006; Young, 1996a 1996b, 1999, 2000; Young \& Rodgers 1998a, 1998b, citados en Castillo, Terol, Nieto, Lledó, Sánchez, Aragón \& Sitges, 2008).

Hasta ahora, no hay una definición concreta de lo que podría considerarse adicción a internet. Sólo se ha llegado a encontrar algunos síntomas característicos, entre los que se destacan preocupación, impulsividad incontrolable, tolerancia, síndrome de abstinencia, uso y esfuerzo excesivo invertido en internet e impedimentos en la habilidad para la toma de decisiones. Varios estudios han utilizado diversos métodos para investigar e identificar las adicciones a internet, $y$ han usado términos como "dependientes de Internet", "usuarios problemáticos de internet" o "usuarios patológicos de internet" (Lin, 2002; Davis, 2001; citados en Tsai, Sunny \& Lin, 2003). La mayoría de estos estudios emplean métodos cualitativos como cuestionarios y entrevistas para evaluar dichas adicciones junto con instrumentos de medición como la tabla de Young (2009), con la cual se puede calcularlas y medirlas.

Cruzado, Matos y Kendall (2006) llevaron a cabo un estudio acerca del perfil de este tipo de adicto y encontraron que el deterioro académico es una conse- 
cuencia del uso excesivo de este medio, a lo que se suman problemas de pareja. Además de que $80 \%$ de sus sujetos adictos tenían una historia de disfunción familiar, también detectaron tendencias al aislamiento social, patrones de evasión de la realidad y otras anomalías tanto conductuales como químicas, como predisposición a la depresión, inestabilidad emocional, pobre tolerancia a la frustración, volubilidad e irritabilidad.

Otros rasgos que se encontraron fueron: egocentrismo, timidez, introversión, baja empatía, baja autoestima, predisposición al aburrimiento, búsqueda de sensaciones, preferencia por actividades solitarias, autosuficiencia, reactividad emocional elevada e inconformidad con las normas sociales. También notaron que estos tipos de rasgos parecen corresponder a personalidades evitativas, dependientes, esquizoides y antisociales.

Un dato muy interesante que encontraron Cruzado et al. (2006) es que los chats ocupan un lugar importante después de los juegos en red respecto de la popularidad online y a la adicción. Los autores refieren que esta característica se debe a que muchos de los adictos experimentan la interacción social como algo amenazante, mientras que Internet es socialmente liberador, porque les facilita la adopción de identidades secretas, con atributos opuestos a los reales, en un entorno de anonimato pero a la vez de intimidad.

El hecho de comunicarse y relacionarse a través de este medio internet supone establecer interacciones en un entorno virtual o ciberespacio. Según Mckenna y Bargh (2000; citados en Toranzo, 2009) existen cuatro características diferenciales fundamentales entre la comunicación cara a cara y la comunicación vía internet: 1) El anonimato, que consiste en la posibilidad de ocultar la verdadera identidad cuando se concreta la comunicación por la red. 2) La ausencia de comunicación no verbal: cuando se lleva a cabo una comunicación por medio de la computadora no hay señales visuales ni auditivas. 3) Distanciamiento físico: en internet la comunicación no está limitada por la dimensión geográfica. 4. Tiempo: este medio permite la existencia de una comunicación en tiempo real o bien retardar las respuestas.

En última instancia, estas características determinan que las relaciones se desarrollen y manifiesten de manera diferente.

\section{INTERNET Y REDES SOCIALES}

El internet se ha expandido por todo el mundo por medio del incremento de su propia información y capacidades, y se ha convertido en una herramienta indispensable para toda la población académica y profesionalmente activa. Esta herramienta se volvió una potente rama de comunicación y hoy en día esta transformación se puede constatar gracias a las redes sociales existentes en línea. (Raacke \& Bonds-Raacke, 2008).

En la actualidad se han realizado estudios más específicos sobre las redes sociales en Internet y su relación con algunos factores sociales. Por ejemplo, se destaca la red social conocida como Facebook, una de las más populares entre los jóvenes hoy en día.

Los sitios como Facebook son relativamente nuevos y su difusión ha traspasado culturas, ya que se considera una nueva forma de comunicación y socialización. Si bien esta comunicación juega un papel muy importante dentro de las nuevas generaciones, también es importante analizar las formas en que produce cambios en las redes sociales físicas y reales (Magnuson \& Dundes, 2008).

Según el psicólogo social Ervin Goffman (citado en Young, 2009), las personalidades virtuales son aquellas que se usan como reflejo del verdadero ser, que pueden idealizarse para agradar a otros. Además, se utilizan muchas poses adoptadas que revelan conformidad y en las que prevalecen códigos tanto masculinos como femeninos.

Los individuos que participan en las redes sociales en línea utilizan el lenguaje, la tecnología y las herramientas multimedia para crearse una identidad. Esto no consiste sólo en tener amigos en línea, sino que también influye en la manera en que se aprende y habilitan necesidades para participar en las nuevas formas de socialización de la era digital. (Young, 2009).

Para que exista adicción a Internet es necesario que se manifiesten varios factores, entre ellos la depresión. Otro elemento de gran importancia es la fobia de ansiedad, ya que a los adolescentes que la padecen no les gusta salir, y la Internet les da la posibilidad de contactarse con otros e involucrarse en juegos sin la necesidad de exponerse a situaciones que les causen angustia.

Dado que aún no hay suficientes estudios acerca del efecto de esta red y su relación con ciertos aspectos, 
en este artículo se tratará de descubrir la problemática de este fenómeno que actualmente incide sobre la población juvenil y su relación con algunos aspectos psicológicos, sociales y emocionales.

\section{FACEBOOK COMO RED SOCIAL}

Como resultado de la popularidad de las redes sociales, sitios como Facebook crecen, al igual que la preocupación sobre el efecto que estos sitios ejercen en el manejo de las impresiones y relaciones que en la actualidad establecen los jóvenes. Buffardi y Campbell, (2008; citado en Strano, 2008) presentan cierta preocupación de que el ambiente en línea genere comportamientos narcisistas, mientras que otros muestran inquietud pues suponen que la permanenecia excesiva en él puede provocar la sustitución de las relaciones de amistad por otras caracterizadas por la superficialidad.

Facebook fue fundado en 2004 como una red social sólo para estudiantes de Harvard, pero a mediados del 2005 fue adoptado por alrededor de otras 2000 universidades y colegios tan sólo en Estados Unidos. A lo largo de este último año, 22,000 organizaciones comerciales se unieron a esta red y a finales de él, dos tercios de usuarios se conectaban a diario y estaban en el sitio un promedio de 20 minutos (Zywica \& Danowski, 2008).

En agosto de 2007 el sitio contaba con 22 millones de usuarios y 15 mil millones de páginas vistas en él (Freiert, 2007; citado en Zywica \& Danowski, 2008).

Zywica y Danowski (2008) explican que Facebook permite a sus usuarios crear perfiles personalizados que incluyen información general, como la educación, el sexo, la edad, etc. Los usuarios pueden escribir y poner mensajes en las páginas de sus contactos, subir fotos y etiquetar personas en esas fotos, además de incluir videos y canciones. Se pueden tener tantos contactos como se quieran, que pueden ser compañeros de escuela, amigos, familiares, conocidos, personas de otros países y continentes, al igual que asociaciones, organizaciones e instituciones.

Mediante diversas investigaciones se ha podido constatar que el empleo que se hace del sitio depende del género de la persona que lo utiliza. Se ha comprobado que las mujeres lo utilizan para dar seguimiento a las relaciones y mantenerse en contacto con amigos, compañeros de trabajo, escuela, familia y demás, mientras que los hombres acceden a él para seguir ciertos impulsos y relaciones de fantasía en las que se puede llegar a obtener alguna gratificación sexual (Raacke \& Bonds-Raacke, 2008).

\section{ADICCIÓN A FACEBOOK}

Diferentes estudios coinciden en señalar que el término "vicio" provoca reacciones afectivas de los adictos que obstaculizan el uso de los servicios de ayuda. En cambio, visualizarlo como enfermedad parece proponer una intervención para solucionarlo. Se ha encontrado que el consumo, visto como vicio, se percibe como algo voluntario, donde la influencia social es lo más importante. La adicción es socialmente vista como algo vergonzoso y rechazado. Dentro de este marco se hace caso omiso de la dependencia, la tolerancia y el síndrome de abstinencia en razón del valor atribuido socialmente a la voluntad. Por otro lado, ver la adicción como una enfermedad es muy útil para poder aceptarla y buscar ayuda. Sin embargo, lo óptimo sería prevenir la adicción, para no tener que tratarla (Nuño, Alvarez, Gónzalez \& Madrigal, 2006).

La descripción de alteraciones comportamentales asociadas a un uso excesivo de la red, tales como sentimientos de culpa, deseo intenso de estar o continuar conectado a ella, pérdida de control y de tiempo de trabajo o de clases, síntomas psicopatológicos, etc., ha abierto un debate sobre una posible "adicción a Internet" ("Trastorno de adicción a Internet", "Uso patológico de Internet", "Uso excesivo de Internet", "Uso problemático de Internet", "Uso compulsivo de Internet"), referido a situaciones en las que el uso de la web puede afectar a diferentes ámbitos de la vida del sujeto (Widyanto \& Griffiths, 2006; citados en Poch, 2009).

Raacke y Bonde-Raacke (2008) llevaron a cabo una investigación en la que descubrieron que $87 \%$ de 116 estudiantes de licenciatura en la costa Este de Estados Unidos tiene una cuenta y perfil de Facebook. Este dato los llevó a destacar la importancia que ha asumido la forma de socialización actual por Internet, sin dejar de lado el hecho de que los estudiantes se sientan más cómodos con estos métodos que con el "cara a cara", lo que puede llegar a repercutir en la socialización per se. En la actualidad existen varios grupos en Facebook llamados "Adicción a Facebook", "I'm a Facebook addict", 
"Are you a Facebook addict?", "Adictos a Facebook!!", "Soy adicto a Facebook y lo acepto", "Maldita sea, soy adicto a Facebook!!!”, entre muchos otros, en los cuales la gente que se considera parte de esos grupos "se hace seguidor" de alguno de ellos.

La cantidad de personas inscritas en estos grupos varía desde 430 hasta 11,890 personas ("Maldita sea, soy adicto a Facebook!!!”).

Por lo general, estos grupos tienen un origen nacional, es decir, sus creadores proceden de varios países como Argentina, México, España, etc.; en ellos existe la posibilidad de que las personas inscritas hagan sus comentarios sobre cualquier tema. Evidentemente, la cantidad de comentarios que se encuentran en estas páginas acerca de las razones que tuvieron sus integrantes para unirse a ellos, las causas de su adicción a Facebook y cómo la justifican también es inmensa.

Dichos grupos pueden hacer hincapié en cuestiones tales como las razones por las cuales pertenecen al grupo, y sobre todo y muy aparte de la misma adicción, problemas como la autoestima, la depresión y las habilidades sociales, que pueden estar implicadas en el uso abusivo de Facebook. Por ello, es de suma importancia poder entender estos términos, así cómo sus definiciones.

\section{AUTOESTIMA}

La autoestima se reconoce como un indicador del desarrollo personal fundado en la valoración, positiva, negativa o neutra, que cada persona hace de sus características cognitivas, físicas y psicológicas. Dicha valoración se construye sobre la base de la opinión que cada persona tiene sobre sí misma, a partir de los atributos que le otorgan las características mencionadas. Esta opinión es confirmada por cada individuo a partir de la percepción de cómo y cuánto lo valora quienes lo rodean y, particularmente, todo aquel que es relevante para él en su vida cotidiana (Arancibia, 1997; Fredes, 1998; citados en Peradotto, Vargas \& Valdivia, 2005). La baja autoestima se relaciona con comportamientos adictivos. Craig (1995; citado en Niemz, Griffiths \& Banyard, 2005), reportó que las personas que efectúan autoevaluaciones negativas utilizan sustancias adictivas para escapar o abstenerse de pensamientos desvalorativos sobre sí mismas. Armstrong, Phillips y Salin (2000), que investigaron si la baja autoestima también estaba relacionada con la adicción a Internet, comprobaron que ella era un buen predictor de dicha adicción, lo que también aumentaba el tiempo que se invertía en línea. Sin embargo, la relación no es clara, ya que, al igual que la depresión, la baja autoestima puede ser una consecuencia de la adicción más que una causa de ella, es decir, que podría provocar el empleo de Internet como una escapatoria, pero también puede suceder que el uso excesivo de la web lleve a las personas a aislarse y después no tengan la misma capacidad para socializar de manera normal. Si las personas tienen una mala opinión de sí mismas y encuentran dificultades para socializar por su timidez o falta de autoestima, pueden usar la Internet como una alternativa de socialización, pues en ella se pueden abrir y ganar confianza sin tener que interactuar cara a cara (Navarro \& Jaimes, 2007).

\section{HABILIDADES SOCIALES}

Las habilidades sociales o competencia social se consideran como una medida sumativa del rendimiento social en las interacciones con iguales y adultos en distintas situaciones, valorado por agentes sociales significativos, que incluyen al propio sujeto (Gresham, 1986; McConnell \& Odom, 1986; citados en Trianes, Blanca, Muñoz, García \& Infante, 2002). La evaluación del comportamiento de ansiedad y fobia social es difícil de llevar a cabo, ya que muchas veces depende de numerosos factores sociales distintos en cada persona. Por eso es importante englobar varios componentes y dimensiones que se consideran en la evaluación (Zubeidat, Sierra \& Salinas, 2007). En 2008 estos investigadores llevaron a cabo un estudio en el cual se analizaron diversos factores asociados con la ansiedad y la fobia social. Estos factores son muy importantes, ya que pueden ser parte de las habilidades sociales; sin embargo, también éstas incluyen muchos factores más. Para realizar el estudio se utilizó una muestra de 1012 adolescentes (con 582 mujeres y 430 hombres), estudiantes de nivel secundaria de la provincia de Granada, España. Los instrumentos de medición fueron subescalas o escalas que miden ansiedad relacionada con distintos factores sociales (escala de ansiedad en interacción social, escala de ansiedad social de Liebowitz, escala de estrés y evitación social y escala de miedo a la evaluación negativa). El trabajo permitió inferir que 
la ansiedad social está asociada directamente con la realización de actividades deportivas, amistad íntima, participación con amigos y el hecho de hacer las cosas por sí mismo. De igual manera, se pudo constatar que las personas que no efectuaban alguna de estas actividades presentaron una ansiedad social mayor que los que sí las llevaban a cabo.

\section{DEPRESIÓN}

En la revista de salud Medline Plus (2008), se define a la depresión como el hecho de sentirse triste, melancólico, infeliz, miserable o derrumbado; además, se menciona que es normal que la mayoría de las personas se sientan de esta manera alguna vez durante cortos periodos de su vida. Sin embargo, la verdadera depresión clínica es un trastorno del estado anímico en el cual los sentimientos de tristeza, pérdida, ira o frustración interfieren con la vida diaria durante un período prolongado. Se habla también de que, por lo general, la baja autoestima es compañera frecuente de la depresión, al igual que los arrebatos repentinos de ira y el hecho de no encontrar placer en actividades que normalmente hacían feliz a la persona que la padece. De acuerdo con los resultados de la investigación llevada a cabo por Tsai, Sunny y Lin (2003), seis de las personas que entrevistaron dijeron que si se sentían de mal humor y deprimidos, se conectaban a Internet para remediar su depresión, para lo cual trataban de hablar con alguien, jugar en línea o hacer "cosas locas" por Internet. Muchos de estos adolescentes manifestaron que la red amplía sus posibilidades de hacer vida social pues en ella conocen gente nueva.

\section{ADICCIÓN A FACEBOOK Y SU RELACIÓN CON LA AUTOESTIMA, LAS HABILIDADES SOCIALES Y LA DEPRESIÓN}

Jiménez y Pantoja (2007) hicieron un estudio acerca de la relación que existe entre la autoestima y las relaciones interpersonales de sujetos adictos a Internet. Estas autoras encontraron que los sujetos adictos presentaban niveles medios y bajos de autoestima. Por el contrario, los niveles del grupo de no adictos eran elevados, lo que las autoras atribuyeron al hecho de tener la capacidad de confiar en sus propios juicios y percepciones, tener actitudes positivas hacia sí mismos y hacia el manejo de eventos críticos así como receptivi- dad a las relaciones e intercambios con los demás. Una de las conclusiones de este trabajo es que las personas que presentaban bajos niveles de autoestima tendían a ser más propensas a desarrollar adicción a Internet $\mathrm{y}$, a su vez, presentaban menos recursos psíquicos disponibles. Por lo tanto, mientras más bajos fueran estos, mayor era la dificultad para establecer relaciones interpersonales significativas y, por consiguiente, mayor también la tendencia a la depresión y el suicidio, En síntesis, los sujetos adictos mostraron menor capacidad de mantener la dirección de su propia conducta y menor habilidad para soportar los aumentos de su tensión psíquica. A diferencia de ellos, los sujetos no adictos se mostraron más equilibrados, y lograban un mayor autocontrol cuando debían hacer frente a elementos estresantes.

En cuanto a las habilidades sociales, comprobaron que $50 \%$ de los sujetos adictos tuvo un mayor número de indicadores de ausencia de habilidades de este tipo, debido a lo cual debían lidiar con una mayor dificultad para manejarse ante la complejidad de la vida cotidiana así como de manejar sus recursos internos.

Jiménez y Pantoja (2007) consideran que la depresión es consecuencia de la dificultad para establecer relaciones interpersonales causada por una baja autoestima; en contraste, McKenna y Bargh (1999; citados en Moral, 2001) descubrieron que las personas no creían que el uso de Internet aumentara su estado de soledad o depresión, sino más bien lo contrario. Al concluir el estudio después de dos años, los datos que obtuvieron McKenna et al. (1999; citados en Moral, 2001) les permitieron determinar que el nivel de soledad había disminuido en $51 \%$ de los sujetos, $43 \%$ no había experimentado efecto sobre la soledad y únicamente $6 \%$ de ellos se sintieron más solos como consecuencia del uso de Internet.

Respecto del grado de depresión experimentada, $2 \%$ respondió que el uso de Internet había aumentado su grado de depresión, $21 \%$ dijo que les había reducido sus sentimientos de depresión, y el resto no reportó ningún efecto sobre la depresión.

En su estudio, McKenna y Bargh (1999; citados en Moral, 2001) informaron que sobre un total de 600 usuarios de Internet entrevistados, $67 \%$ contestó que su círculo social había aumentado, y sólo 4\% informó, a diferencia de otros estudios, que tenía menos conoci- 
dos y amigos como resultado de usar Internet. De igual manera, descubrieron que el desarrollo y la identificación de las personas con un grupo de discusión en el que participan y comparten un mismo tema de interés (situaciones encontradas en Internet), ejercen un papel positivo en su autoestima personal.

Todos los factores mencionados logran crear preguntas y cuestiones que son de suma importancia en este tema, por ejemplo: ¿Está relacionada la adicción a Facebook con la depresión? ¿la adicción a Facebook tiene alguna relación con la falta de competencia social?, o ¿se relaciona con una baja autoestima? Dada toda la información encontrada, los estudios previos sobre la adicción a Internet y el efecto de este tema en nuestra vida actual, en este artículo se pretenderá encontrar si existe la adicción a Facebook, y de ser así, en qué niveles y cómo se relaciona con las variables de depresión, falta de competencia social (habilidades sociales) y baja autoestima.

\section{MÉTODO}

Para llevar a cabo la investigación se utilizó un diseño no experimental de tipo transversal, en el cual se observó el efecto de la variable independiente sobre la dependiente. Se trata de una investigación ex post facto, ya que se observaron efectos de eventos ya ocurridos.

\section{Participantes}

Para esta investigación se seleccionó un total de 60 participantes, todos estudiantes a nivel licenciatura, que se distribuyeron en dos grupos: adictos a Facebook $(\eta=30)$ y no adictos a Facebook $(\boldsymbol{\eta}=30)$. Por su parte, para hacer una clasificación de adictos y no adictos, dentro del cuestionario se incluyó una escala de adicción: los que reportaron que invertían más de 4 horas diarias conectados fueron clasificados como adictos. Todos los sujetos, que fueron seleccionados de manera no aleatoria, eran estudiantes de Licenciatura de la Universidad Iberoamericana campus Santa Fe. Se tomaron en cuenta factores tales como el nivel socioeconómico, la ocupación, la escolaridad, el lugar de procedencia, la nacionalidad, la edad, el estado civil, el hecho de tener una pareja, etc. Como factor muy importante se consideró el lugar o lugares donde podían acceder a Internet, tales como la casa, el trabajo, la escuela, una laptop personal, el celular, entre otros.
Al correr los análisis estadísticos, se puede observar que de los 60 participantes, la edad de mayor frecuencia es 22 años, mientras que la media es de 21.49 años. En el momento de aplicárseles los instrumentos, $98.4 \%$ de ellos eran solteros; $38.1 \%$ no tenía pareja, mientras que el resto sí la tenía. Se detectó que la carrera que con mayor frecuencia cursaban era la de Psicología, con un porcentaje de 52.4, seguida por la de Comunicación con un porcentaje de 20.6; la tercera era Arquitectura con 6.3\%; en cuarto lugar, Administración de la Hospitalidad con $4.8 \%$; en quinto lugar se encontraban las carreras de Historia del Arte con 3.2\%, Mercadotecnia con 3.2\% y Administración de Empresas con 3.2\%. En sexto lugar se encontraban las carreras de Diseño Gráfico, Ingeniería Civil, Pedagogía y Finanzas, todas con un porcentaje de 1.6. Respecto de los años de estudio, 34.9\% (el más alto) tenía 19 años cursados de estudio y el más bajo-16 años- correspondía a 4.8\%. En cuanto al sexo, $60.3 \%$ pertenecía al género femenino y $39.7 \%$ al masculino.

\section{Instrumentos}

A) La variable independiente adicción a Facebook se midió con El Cuestionario de Adicción a Facebook para jóvenes de propia creación (2009), basado en Los Criterios de Diagnóstico de Adicción a Internet propuestos por Young (1998; citado en Navarro, 2007) y en El Cuestionario de Experiencias Relacionadas con Internet (CERI), creado por Marta Beranuy Fargues, Andrés Chamarro Lusar, Carla Graner Jordania y Xavier Carbonell Sánchez (2009), el cual evalúa los conflictos intra e interpersonales relacionados con el uso de Internet. El CERI consta de una escala de respuesta tipo Likert de cuatro puntos. La escala I-6 mostró una consistencia interna de 0.63 y la M-6 de 0.71 . El primer factor (conflictos intrapersonales) incluyó 6 ítems que explicaron $27.14 \%$ de la varianza; el segundo (conflictos interpersonales) incluyó 4 ítems que daban cuenta de $12.18 \%$ de la varianza. El primer factor mostraba una consistencia interna de 0.74 y el segundo de 0.75 . El conjunto de la escala mostraba una consistencia interna de 0.77 .

El Cuestionario de Adicción a Facebook para jóvenes creado para detectar si existe o no éste tipo de adicción y el nivel de ella consta de dieciocho reactivos en escala Likert con las opciones de respuesta: nunca, 
casi nunca, casi siempre y siempre; siete reactivos con respuesta abierta, tres reactivos en escala Likert con las opciones de respuesta: diario, 6 a 4 veces a la semana, 3 a 1 veces a la semana, 0 veces, un reactivo con las opciones de respuesta: Sí, No, y finalmente un reactivo en escala Likert con las opciones de respuesta: cibercafé, laptop, computadora en casa, celular y otro. Todo ellos suman treinta reactivos en total.

B) La variable dependiente autoestima se midió con el Cuestionario de evaluación de la Autoestima para alumnos de enseñanza secundaria (Gómez, 1998), que consta de diecinueve ítems, dieciséis de los cuales se referían a cualidades propias de la autoestima y tres a una escala de autocrítica que evaluó en qué medida los alumnos contestaron o no influidos por los efectos de la deseabilidad social.

Este cuestionario presenta una estructura factorial, compuesta por: Autoestima física, Autoestima general, Autoestima de competencia académico/intelectual, Autoestima emocional y Autoestima de relaciones con los demás.

El índice de autoestima general supone la puntuación de mayor relieve de todo el cuestionario, ya que reflejaba el nivel global de autoestima de los alumnos. Este índice de la puntuación total representa la suma de las puntuaciones de todas las dimensiones del autoconcepto, sin tomar en consideración las puntuaciones de la escala independiente de autocrítica.

La prueba arrojó un coeficiente alpha de fiabilidad de 0,70. Mediante el procedimiento de las dos mitades, a través de la fórmula ideada por Spearman Browm, también se obtiene un coeficiente de fiabilidad de 0,70.

C) La variable dependiente habilidades sociales se midió con el Cuestionario de Evaluación de Dificultades Interpersonales en la Adolescencia (CEDIA), creado por Cándido, Inglés Saura, Méndez e Hidalgo (2000), destinado a detectar el nivel de dificultad que presentan los adolescentes (de 13 a 19 años) en sus relaciones interpersonales. Esta herramienta está compuesta por 39 ítems distribuidos en cinco factores: Aserción, Relaciones con el otro Sexo, Relaciones con Iguales, Hablar en Público y Relaciones Familiares, que explican $32.92 \%$ de la varianza. El estudio de la fiabilidad indica que el CEDIA obtuvo una elevada consistencia interna $(\mathrm{a}=.91)$. En la composición de acuerdo con cada uno de los factores se observa que Aserción, que explica $9.86 \%$ de la varianza total, incluyó 12 ítems relativos a las relaciones de los adolescentes con personal de servicios (camareros y dependientes); Relaciones con el otro Sexo, que dio cuenta de $6.21 \%$ de la varianza, incluyó 7 ítems; Relaciones con Iguales, que explicó $5.95 \%$ de la varianza, se componía de 9 ítems; Hablar en Público explicó el 5.73\% de varianza y comprendió 6 ítems; Relaciones Familiares, y relaciones de no iguales, que explicó $5.17 \%$ de varianza, estaba compuesto por 6 ítems.

D) La variable dependiente depresión se midió con La Escala de Depresión Geriátrica (GDS), creada por Yesavage (1986), probada y usada extensamente con poblaciones de adultos mayores. El cuestionario largo GDS es una herramienta breve de 30 puntos en el que los participantes deben responder sí o no con respecto a cómo se sintieron en la última semana. En 1986 se creó un cuestionario corto GDS, que consta de 15 preguntas.

Para la versión corta (que fue la que se utilizó en este estudio), se seleccionaron las preguntas del cuestionario largo GDS que guardaban la mayor correlación con los síntomas depresivos en los estudios de validación. De los 15 puntos, 10 indicaban la presencia de depresión cuando se contestaban afirmativamente, mientras que el resto (preguntas número 1, 5, 7, 11 y 13) la indicaban cuando se respondían negativamente. El GDS se ha usado en adultos mayores con buena salud, con enfermedad médica y en aquellos con deterioro cognitivo de leve a moderado. También se ha aplicado extensamente en entornos comunitarios, de atención de agudos síntomas y de cuidados de largo plazo.

La sensibilidad del GDS fue de $92 \%$ y la especificidad de $89 \%$ cuando la evaluación se realizó con criterios diagnósticos. La validez y confiabilidad de la herramienta han sido respaldadas tanto con la consulta como con la investigación clínica. En un estudio de validación que comparaba los cuestionarios corto y largo de GDS para la autocalificación de los síntomas de la depresión, ambos cumplieron su objetivo para diferenciar entre adultos deprimidos y no deprimidos con una correlación alta $(r=0.84, p<0.001)$ (Yesavage, 1986; citado en De la Vega, 2009). 


\section{Procedimiento}

A los 63 participantes se les aplicó un cuestionario en un periodo de una semana, cada uno de los cuales incluía escalas de adicción a Internet, adicción a Facebook, depresión, autoestima y habilidades sociales. El cuestionario se aplicó a alumnos de una universidad privada del Distrito Federal, los cuales fueron escogidos de manera aleatoria y voluntaria. Previamente se les preguntó si se consideraban adictos a Internet y a Facebook o no y dependiendo de sus respuestas se aplicó el cuestionario a las personas que reportaban que eran adictos a Facebook y no a Internet, así como a los que contestaron que no eran adictos a ninguno de los dos. A los que contestaron que eran adictos a ambos se les aplicó preguntándoles para qué usaban Internet: si contestaban que lo utilizaban para revisar el mail o hacer tareas se les pidió que pusieran que lo usaban con fines escolares para evitar la variable extraña de adicción a Internet. Por último, a los que contestaron que eran adictos a Internet pero no a Facebook no se les aplicó el cuestionario. Al final se juntaron todos los cuestionarios y se dividieron con base en los dos grupos "adictos" y "no adictos" a Facebook, para poder compararlos y analizar los datos para obtener los resultados.

\section{RESULTADOS}

Para saber si existían diferencias estadísticamente significativas entre el grupo de adictos a Facebook y el de los no adictos respecto de la relación de las variables de la adicción a Facebook con la baja autoestima, la depresión y la falta de habilidades sociales se aplicó la prueba de muestras independientes t de Student.

Tabla 1. Comparación de las diferencias estadísticamente significativas entre el grupo de adictos a Facebook y el grupo de los no adictos, respecto de la relación de las variables de la adicción a Facebook con baja autoestima, depresión y falta de habilidades sociales.

\begin{tabular}{|c|c|c|c|c|}
\hline Factor o variable & Grupos & Medias & $\mathrm{t}$ & Significancia \\
\hline Depresión & $\begin{array}{c}\mathbf{1}=\text { No adictos a Facebook } \\
\mathbf{2}=\text { Adictos a Facebook }\end{array}$ & $\begin{array}{l}1.40 \\
4.53\end{array}$ & 5.635 & .000 \\
\hline $\begin{array}{l}1^{\circ} \text { factor (habilidades sociales) } \\
\text { Aserción }\end{array}$ & $\begin{array}{c}\mathbf{1}=\text { No adictos a Facebook } \\
\mathbf{2}=\text { Adictos a Facebook }\end{array}$ & $\begin{array}{l}.73 \\
1.40\end{array}$ & 4.399 & .000 \\
\hline $\begin{array}{l}2^{\circ} \text { factor (habilidades sociales) } \\
\text { Relaciones con Iguales }\end{array}$ & $\begin{array}{c}\text { 1=No adictos a Facebook } \\
\mathbf{2}=\text { Adictos a Facebook }\end{array}$ & $\begin{array}{c}.44 \\
1.13\end{array}$ & 4.078 & .000 \\
\hline $\begin{array}{l}3^{\circ} \text { factor (habilidades sociales) } \\
\text { Hablar en Público }\end{array}$ & $\begin{array}{c}\mathbf{1}=\text { No adictos a Facebook } \\
\mathbf{2}=\text { Adictos a Facebook }\end{array}$ & $\begin{array}{c}.89 \\
1.65\end{array}$ & 4.457 & .000 \\
\hline $\begin{array}{l}4^{\circ} \text { factor (habilidades sociales) } \\
\text { Relaciones Familiares }\end{array}$ & $\begin{array}{c}\text { 1=No adictos a Facebook } \\
\mathbf{2}=\text { Adictos a Facebook }\end{array}$ & $\begin{array}{c}.83 \\
1.25\end{array}$ & 2.040 & .047 \\
\hline $\begin{array}{l}5^{\circ} \text { factor (autoestima) } \\
\text { Autoestima Física }\end{array}$ & $\begin{array}{c}\text { 1=No adictos a Facebook } \\
\mathbf{2}=\text { Adictos a Facebook }\end{array}$ & $\begin{array}{l}12.81 \\
10.65\end{array}$ & 3.799 & .000 \\
\hline $\begin{array}{l}6^{\circ} \text { factor (autoestima) } \\
\text { Autoestima General }\end{array}$ & $\begin{array}{c}\mathbf{1}=\text { No adictos a Facebook } \\
\mathbf{2}=\text { Adictos a Facebook }\end{array}$ & $\begin{array}{l}17.40 \\
14.48\end{array}$ & 4.116 & .000 \\
\hline $\begin{array}{l}7^{\circ} \text { factor (autoestima) } \\
\text { Autoestima de competencia académico/ } \\
\text { intelectual }\end{array}$ & $\begin{array}{c}\mathbf{1}=\text { No adictos a Facebook } \\
\mathbf{2}=\text { Adictos a Facebook }\end{array}$ & $\begin{array}{l}9.96 \\
8.62\end{array}$ & 2.916 & .005 \\
\hline $\begin{array}{l}8^{\circ} \text { factor (autoestima) } \\
\text { Autoestima Emocional }\end{array}$ & $\begin{array}{c}\mathbf{1}=\text { No adictos a Facebook } \\
\mathbf{2}=\text { Adictos a Facebook }\end{array}$ & $\begin{array}{l}7.12 \\
5.93\end{array}$ & 2.332 & .023 \\
\hline $\begin{array}{l}9^{\circ} \text { factor (autoestima) } \\
\text { Autoconcepto en general }\end{array}$ & $\begin{array}{c}\mathbf{1}=\text { No adictos a Facebook } \\
\mathbf{2}=\text { Adictos a Facebook }\end{array}$ & $\begin{array}{l}57.12 \\
48.68\end{array}$ & 4.659 & .000 \\
\hline
\end{tabular}


Como se puede observar a partir de la media de los primeros cinco factores [Depresión, Aserción, Relaciones con Iguales, Hablar en Público y Relaciones Familiares (los últimos 4 factores se refieren a habilidades sociales)], los no adictos a Facebook (grupo 1), obtuvieron puntajes más bajos, mientras que los adictos (grupo 2) obtuvieron puntuaciones más altas.

Es significativo que los adictos a Facebook son más propensos a la depresión, a ser menos asertivos, a tener menos calidad de relaciones tanto con amigos como con la familia, o a tener más dificultades para hablar en público que los no adictos ( $\mathrm{t}=5.5001 ; \mathrm{p}=.001$ ). Asimismo, se observa que en la media de los últimos cinco factores: Autoestima Física, Autoestima General, Autoestima de competencia académico/ intelectual, Autoestima Emocional, Autoestima de relaciones con los demás y Autoconcepto en general (todos éstos son factores de autoestima), los no adictos a Facebook (grupo 1) puntuaron más alto, mientras que los adictos (grupo 2) tuvieron puntuaciones menores, ya que los valores fueron codificados de manera inversa.

También es significativo que los adictos a Facebook tienen una mayor tendencia a la baja autoestima. Por ello, muestran una percepción más devaluada de su físico, de su autoconcepto y de su intelecto, al igual que una percepción deteriorada ante determinadas situaciones que pueden provocar estrés en sus relaciones interpersonales; además, tienen menos defensas, es decir, son sujetos patológicamente indefensos ( $\mathrm{t}=5.5001 ; \mathrm{p}=.001)$. Los no adictos (grupo 1), presentaron menores puntajes en las variables depresión, (media de 1.41) y relaciones familiares (media de 0.839), y mayores puntajes en las variables autoestima general $($ Media $=17.41)$, y autoestima total $($ Media $=57.13)$. Por otro lado, los adictos (grupo 2), presentaron menores puntajes en las variables autoestima general (Media=14.48) y autoestima total $($ Media $=48.69)$; $\mathrm{y}$ mayores puntajes en las variables depresión $($ Media $=4.54)$, y relaciones familiares (Media= 1.253).

Esto quiere decir que, en relación con los no adictos, los que sí lo son tienen una mayor propensión a la depresión, no tienen relaciones familiares tan buenas y presentan menor nivel de autoestima general y total. En resumen, se comprobó una relación estadísticamente significativa entre la adicción a Facebook y la baja autoestima, la depresión y falta de habilidades sociales.

\section{DISCUSIÓN}

El uso de Internet y de las redes sociales, como Facebook, es un tema que tiene que empezar a ser estudiado de manera meticulosa, ya que cada día saltan al escenario de la web incontables usuarios que tienen un tipo de cuenta dentro de ellas. Hoy en día, estos medios de comunicación son utilizados de manera frecuente por estudiantes universitarios, lo cual invariablemente cambia según la cultura y el país, y depende del acceso a Internet, el número de horas de tiempo libre y otros factores. Por ejemplo, 53\% de la población de Canadá, tiene un perfil activo en Facebook, lo cual lo convierte en el país con mayor acceso diario. En contraste, Alemania sólo cuenta con $17 \%$ de su población activa (Young, 2009). En el caso de México no se ha encontrado información que se pueda comparar.

El uso cada vez mayor de las redes sociales electrónicas ha sido objeto de distintos estudios, como se puede apreciar en la investigación que se llevó a cabo en el año 2007 en la que de una muestra de 116 estudiantes de licenciatura (53 hombres y 63 mujeres), $87.1 \%$ de los sujetos contaba con acceso como usuario a redes sociales, y de ese $87.1 \%, 90.1 \%$ reportó contar con una cuenta de Facebook (Raacke \& Bonds-Raacke, 2008). De acuerdo con un estudio que realizó la investigadora mexicana Guadalupe Victorica en 2004 con adolescentes de 15 a 19 años en la ciudad de Monterrey, la mitad de los consultados dijo que dedicaba de una a cinco horas a la semana a navegar en Internet.

Es importante definir que un adicto en general es una persona que presenta inhabilidad social o menor habilidad para soportar los aumentos de su tensión psíquica. En contraste, los sujetos no adictos son más equilibrados y logran un mayor control de sí mismo cuando están frente a elementos estresantes tanto internos como externos (Jiménez \& Pantoja, 2007). En la presente investigación se consideró que los adictos a Facebook son aquellos sujetos que pasan más de 4 horas diarias en dicha red, por que se observó que muchas de las horas en que los estudiantes están conectados a Internet se encuentran interactuando dentro de redes sociales.

Por otro lado, McKenna y Bargh (1999; citados en Moral, 2001) descubrieron que de un total de 600 usuarios de Internet que utilizan redes sociales en línea, $67 \%$ aumentó su círculo social gracias a estos medios 
de comunicación, y sólo 4\% informó que tenían menos conocidos y amigos como resultado de usarlos. A diferencia del presente estudio, se encontró que el desarrollo y la identificación de las personas con un grupo de discusión, en el que participan y donde comparten un mismo tema de interés (situaciones encontradas en Internet), ejercen un papel positivo en su autoestima personal.

Cabe recalcar que el hecho de pasar más tiempo dentro de las redes sociales (como Facebook), no significa que las personas aumenten su interacción social, ni su autoestima y ni que obtengan mayor satisfacción en sus relaciones, pues el elemento esencial de la socialización y del proceso de comunicación se encuentra en la calidad de las interacciones del sujeto, así como en su personalidad y en la capacidad que tiene para captar la del otro (Rivero, 2005). Así se pueden entender los datos que obtuvo otro estudio en el cual se puntualizó el hecho de que la autoestima es menor en los usuarios patológicos. Esto se debe a que puede estar relacionada con los efectos desinhibitorios; es decir, que si una persona tiene una opinión baja de sí misma y encuentra dificultades para socializar por su timidez o falta de autoestima, puede usar Internet como una alternativa de socialización para lograr abrirse y ganar confianza sin tener que interactuar cara a cara (Niemz, et al., 2005). Estos resultados pueden ser comparados con nuestra investigación, ya que las personas que obtuvieron un resultado de adicción a Internet, obtuvieron puntaciones que los clasificaban dentro de clases de baja autoestima, y por lo tanto intentan conectarse a estas redes porque les dan seguridad.

Jiménez y Pantoja (2007) descubrieron que las personas que presentan bajos niveles de autoestima tienden a ser más propensas a desarrollar adicción a Internet, a la vez que presentan menos recursos disponibles $y$, por lo tanto, mientras más bajos son éstos, mayor es la dificultad que tienen para establecer relaciones interpersonales significativas y, por consiguiente, también es mayor su tendencia a la depresión y al suicidio. En el presente estudio se comprobó que la autoestima general de los 63 sujetos era considerablemente más baja en el grupo de personas adictas a Facebook, lo que concuerda con los resultados del estudio citado.

Con respecto a la relación entre el empleo de Internet y la depresión, $2 \%$ de los sujetos respondió que el uso de este medio había aumentado su grado de depresión, $21 \%$ dijo que les había reducido sus sentimientos de depresión; mientras que el resto no informó de ningún efecto del uso de Internet sobre esta afección (Moral, 2001). Sin embargo, en la presente investigación se pudo observar que las personas que presentan adicción a Facebook tienden a presentar un nivel mayor de depresión que las no adictas a esta red.

Es importante tomar en cuenta la relación entre el factor depresión con la adicción a Internet, por lo cual se sugiere que se debe intervenir e investigar el efecto que este padecimiento ejerce sobre las personas que pasan un tiempo considerable conectadas. También se asoció a esta adicción la fobia de ansiedad, ya que a los adolescentes que padecen dicha fobia no les gusta salir, y la Internet les da la posibilidad de contactar a otros e involucrarse en juegos y formas de comunicación sin necesidad de exponerse a situaciones que les provocan angustia (Yen, Ko, Yen, Chen, Chung \& Chen, 2008).

Las fobias y la ansiedad social que sufren muchas personas adictas a Internet pueden ser consideradas como habilidades sociales, tales como la aserción, las relaciones entre iguales, hablar en público y las relaciones familiares; en los datos recolectados por este estudio se pudo observar que todos estos factores eran significativamente más altos en los sujetos que presentan una adicción a Facebook que en los sujetos no adictos a esta red social.

Por último, es importante mencionar que existen personas propensas a sufrir trastornos de personalidad que pueden reflejarse en el uso adictivo de estas redes sociales, por lo que se propone una futura investigación en la que se trate de medir los tipos de personalidades más propensos a abusar de este medio de comunicación y socialización; luego, a partir de este tipo de personalidad, entender qué uso se le da al sitio, para más tarde poder desarrollar nuevas herramientas que contrarresten la adicción y estimulen el buen manejo de las redes. 


\section{REFERENCIAS}

Armstrong, L., Phillips, J. G. \& Saling, L. L. (2000). Potential determinants of heavier Internet usage. International Journal of Human-Computer Studies, 53, 4, 537-550.

Cándido, J., Saura, I., Méndez, F. \& Hidalgo, D. (2000) Cuestionario de Evaluación de Dificultades Interpersonales en la Adolescencia (CEDIA). Psicothema, 21, 3, 480- 485 .

Castillo, J. A., Terol, M. C., Nieto, M., Lledó A., Sánchez, S., Aragón M. \& Sitges E. (2008). Uso y abuso de Internet en jóvenes universitarios. Psiquiatría Interactiva, 20, 2, 131-142.

Cruzado, L., Matos, L. \& Kendall, R. (2006). Adicción a Internet: Perfil clínico y epidemiológico de pacientes hospitalizados en un instituto nacional de salud mental. Revista Médica Herediana, 17(4), 196-205.

De la Vega, R. (4 de septiembre de 2009). Circunvalación del hipocampo. Recuperado el 20 de septiembre de 2009, de http://www.hipocampo.org/yesavagepetite. asp.

Fargues, M., Lusar, A., Jordania, C. \& Sánchez, X. (2009). Validación de dos escalas breves para evaluar la adicción a Internet y el abuso del móvil. Psicothema, 21 (3), 480- 485.

García, A. (1998). Programa para la mejora de las habilidades sociales y la autoestima. Premios Joaquín Sama a la Innovación Educativa. Junta de Extremadura.

Jiménez, L. \& Pantoja, A. V. (2007). Autoestima y relaciones Interpersonales en sujetos adictos a Internet. Psicología-Segunda Época, 26(1), 78- 89.

Llamas, S. (2005). ¿Existe la adicción a Internet? Revista Profesional Española de Terapia Cognitivo-Conductual, 3, 71- 93.

Magnuson, M. \& Dundes, L. (2008). Gender differences in "Social portratis" reflected in MySpace profiles. Cyberpsychology \& Behavior, 11(2), 239-241.

Medline, Plus. Recuperado el 20 de septiembre de 2009, de http://www.nlm.nih.gov/medlineplus/spanish/ency/ article/003213.htm.

Moral, F. (2001). Aspectos psicosociales de la comunicación y de las relaciones personales en Internet. Revista Anuario de Psicología, 32 (2), 13- 30.

Navarro, A. \& Jaimes, G. (2007). Adicción a Internet, revisión crítica de la literatura. Revista Colombiana de Psiquiatría, 26 (4), 691- 700.
Niemz, K., Griffiths, M. \& Banyard, P. (2005). Prevalence of pathological Internet use among university students and correlations with self-esteem, the general health qustionaire (GHQ), and disinhibition. CyberPsychology \& Behavior, 8 (6), 562-570.

Nuño, B. Alvarez, J., Gónzalez, C. \& Madrigal, E. (2006). La adicción, ¿Vicio o enfermedad? Imágenes y uso de servicios de salud en adolescentes usuarios y sus padres. Salud mental, 29 (4) 47-54.

Peradotto, P., Vargas, J. \& Valdivia, R. (2005). Autoestima y refuerzo en estudiantes de $5^{\circ}$ básico de una escuela de alto riesgo. Revista anales de psicología, 21 (1), 102- 115 .

Poch, F. (2009). Uso autoinformado de Internet en adolescentes: Perfil psicológico de un uso elevado de red. International Journal of Psychology \& Psychological Therapy, 9 (1), 109-122.

Raacke, J. \& Bonds-Raacke, J. (2008). MySpace and Facebook: Applying the uses and gratifications theory to exploring friend-networking sites. Cyberpsychology \& Behavior, 11 (2), 169-174.

Rivero, G. (2005). Algunas caracteristícas de la comunicación entre jóvenes y adolescentes en el inicio de relaciones interpersonales en la comunidad. Revista Cubana de Psicología, 22 (1), 68-70.

Strano, M. (2008). User Descriptions and Interpretations of Self-Presentation through Facebook Profile Images. Cyberpsychology, 2 (2), 1-11.

Toranzo, F. (2009). Internet como marco de comunicación e interacción social. Comunicar, 17(32), 231-237.

Trianes, M., Blanca M. J., Muñoz, A., García M. \& Infante L. (2002). Relaciones entre evaluadores de la competencia social en preadolescentes: Profesores, iguales y autoinformes. Revista anales de psicología, 18 (2), 197- 214.

Tsai, C., Sunny, S.J. \& Lin, A. (2003). Internet Addiction of Adolescents in Taiwan: An Interview Study. CyberPsychology \& Behavior, 6(6), 649-652.

Yen, J., Ko, C., Yen, C., Chen, S., Chung, W., \& Chen, C. (2008). Psychiatric symptoms in adolescents with Internet addiction: Comparison with substance use. Psychiatry \& Clinical Neurosciences, 62 (1), 9-16.

Young, K. (2009). Online Social Networking: An Australian Perspective. International Journal of Emerging Technologies \& Society, 7 (1), 39-57. 
Zermeño, A., Arellano, A. \& Ramírez, V. (2005). Redes semánticas naturales: técnica para representar los significados que los jóvenes tienen sobre la televisión, Internet y expectativas de vida. Estudios sobre las culturas contemporáneas, 11 (22), 305-334.

Zubeidat, I., Salinas, J., \& Sierra, J. (2008). Evaluación de factores asociados a la ansiedad social y a otras psicopatologías en adolescentes, Clínica y salud. 31 (3), 189-196.
Zywica, J., \& Danowski, J. (2008). The faces of Facebookers: Investigating social enhancement and social compensation hypotheses; predicting Facebook and offline popularity from sociability and self-esteem, and mapping the meanings of popularity with semantic networks. Journal of Computer-Mediated Communication, 14, 1-34. 